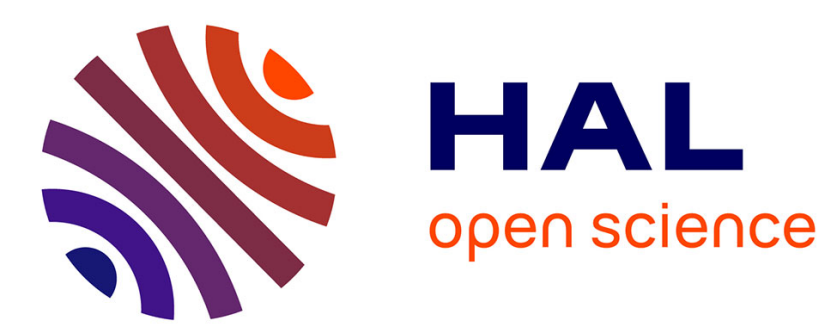

\title{
Molecular Simulations of Hugoniots of detonation products mixtures at chemical equilibrium: Microscopic calculation of the Chapman-Jouguet State
}

Emeric Bourasseau, Vincent Dubois, Nicolas Desbiens, Jean-Bernard Maillet

\section{- To cite this version:}

Emeric Bourasseau, Vincent Dubois, Nicolas Desbiens, Jean-Bernard Maillet. Molecular Simulations of Hugoniots of detonation products mixtures at chemical equilibrium: Microscopic calculation of the Chapman-Jouguet State. Journal of Chemical Physics, 2007, 127, pp.084513. 10.1063/1.2766939 . hal-00143900

\author{
HAL Id: hal-00143900 \\ https://hal.science/hal-00143900
}

Submitted on 3 Nov 2007

HAL is a multi-disciplinary open access archive for the deposit and dissemination of scientific research documents, whether they are published or not. The documents may come from teaching and research institutions in France or abroad, or from public or private research centers.
L'archive ouverte pluridisciplinaire HAL, est destinée au dépôt et à la diffusion de documents scientifiques de niveau recherche, publiés ou non, émanant des établissements d'enseignement et de recherche français ou étrangers, des laboratoires publics ou privés. 


\title{
Molecular simulations of Hugoniots of detonation products mixtures at chemical equilibrium: Microscopic calculation of the Chapman-Jouguet State
}

\author{
Emeric Bourasseau ${ }^{\dagger *}$, Vincent Dubois ${ }^{\star}$, \\ Nicolas Desbiens ${ }^{\dagger}$, Jean-Bernard Maillet ${ }^{\dagger}$ \\ ${ }^{\dagger}$ CEA, Département de Physique Théorique et Appliquée \\ ${ }^{\star} C E A, D I F$ \\ BP12, 91680 Bruyères-le-Châtel, France
}

\begin{abstract}
In this work, we used simultaneously the Reaction Ensemble Monte Carlo (ReMC) method and the Adaptive Erpenbeck Equation Of State (AE-EOS) method to directly calculate the thermodynamical and chemical equilibrium of mixtures of detonation products on the Hugoniot curve. The ReMC method (W. R. Smith and B. Triska, J. Chem. Phys. 100, pp 3019-3027 (1994)) allows to reach the chemical equilibrium of a reacting mixture, and the AE-EOS method (J. J. Erpenbeck, Phys. Rev. A, 46, p 6406 (1992)) constrains the system to satisfy the Hugoniot relation. Once the Hugoniot curve of the detonation products mixture is established, the CJ state of the explosive can be determined. Performing a NPT simulation at $\mathrm{P}_{C J}, \mathrm{~T}_{C J}$, we then calculate the direct thermodynamic properties and the following derivative properties of the system using a fluctuation method: calorific capacities, sound velocity and Grüneisen coefficient. As the composition fluctuates, and the number of particles is not necessarily constant in this ensemble, a fluctuation formula has been developed to take into account the fluctuations of mole number and composition. This type of calculation has been applied to several usual energetic materials: nitromethane, tetranitromethane, hexanitroethane, PETN and RDX.
\end{abstract}

\section{INTRODUCTION}

In the classical description of ideal onedimensional planar detonation waves using the ZND model, the propagation of the wave in the explosive is associated with a sudden increase of pressure and temperature which brings the system onto a particular point of its hugoniot curve: the ZND state. At this point (high pressure and temperature), chemical reactions start to occur, and bring the system from the ZND state (on the hugoniot of the inert explosive) to a point on the hugoniot of the mixture of detonation products, the Crussard curve. Only one point of this curve could support a stationary behavior of the reactive wave, the

*corresponding author: emeric.bourasseau@cea.fr
Chapman-Jouguet state, where the entire system is supposed to be reacted and at chemical equilibrium. From this point, the system then expands isentropically, with a decrease of both temperature and pressure. As a consequence, the calculation of the Crussard curve and the CJ point is particularly important, because it characterizes the initial state of the system after the detonation, before the isentropic expansion. The Crussard curve and the CJ point can be calculated using thermochemical methods through perturbation theories and simplified models. These methods can use a wide variety of equations of state either empirical or theoretical. Thus, the MCRSR method [1] is a standard method in the literature and allows nowadays to correctly reproduce the detonation properties of a variety of explosives [2]. This method is based on a variational 
theory using molecular interactions described by exponential-6 potential models. It is also possible to treat a mixture of several different molecules by reducing the mixture to an equivalent pure fluid using the Ree's mixture law in the so-called one-fluid approximation [3]. Nevertheless, the use of such methods necessarily implies several approximations concerning theoretical background as well as potential models, which make the method less accurate and transferable than molecular simulations.

In this work, we then propose a method to calculate the Crussard curve and the CJ state through molecular Monte Carlo simulations. This presents two main advantages. In the first hand, the use of statistical thermodynamics ensures that the exact EOS of the model material is obtained from atomistic simulations without any approximation. In a second hand, the Monte Carlo method allows to perform simulations using a large variety of potential models from simple, mono center LennardJones materials, to complex, fully atomistic molecular systems (including all atoms models, fluctuating charges, ...), leading to more accuracy and transferability. In this paper, the method is tested with very simple potential models, where molecules are represented by single exponential-6 force centers, in the same fashion as in our thermochemical code [2]. This allows a direct comparison of our results with those coming from thermochemical codes. Results concerning more accurate simulations performed with more complex potential models will be published later.

In this paper, we describe the two methods (ReMC and AE-EOS) used simultaneously to obtain the Crussard curve of any given energetic material. The first method is the Reaction Ensemble Monte Carlo (ReMC) method. This method allows to reach the thermodynamical and chemical equilibrium of a molecular system constrained by chemical equations. This method has been proposed at the same time by Smith and Triska [4] and Johnson et al. [5]. The second method is the Adaptive Erpenbeck Equation Of State (AE-EOS) method, proposed by Erpenbeck [6] and adapted by Brennan and Rice [7]. This method constrains the system to converge toward a point on its hugoniot curve where the Rankine-Hugoniot relations are satisfied. The simultaneous use of both methods has been applied to simple systems constrained by only one chemical equation [7]. In this work, we have coupled the ReMC method implying several chemical equations and the AE-EOS method to perform simulations of realistic mixtures of detonation products composed of up to 7 different molecular species implied in up to 3 simultaneous chemical equilibrium.

Using this method, we have calculated the Crussard curve of 5 usual energetic materials: tetranitromethane (TNM), hexanitroethane (HNE), PETN, RDX and nitromethane (NiMe). Then, the CJ states of those five systems have been determined and compared to experimental CJ properties and results obtained from thermochemical calculations.

In a third time, we have performed Reaction Ensemble NPT simulations at the CJ conditions, in order to obtain thermodynamic derivative properties of the detonation products mixtures at the CJ point. Indeed, those quantities are known to play an important role in the behavior of the detonating system. More precisely, the calorific capacity at constant pressure, the sound velocity and the Grüneisen coefficient are needed for both inert explosive and detonation products mixture in order to feed hydrodynamic codes. Unfortunately, it is also very difficult to obtain those quantities by experimental measurements. Indeed, experiments needed to obtain derivative properties in suitable thermodynamic conditions are often too expensive and too complicated to be performed. Monte Carlo methods appear particularly well suited to obtain derivative properties because fluctuations formula can be 
used to obtain the main derivative properties through a single simulation. Lagache et al., for instance, have derived the expressions to obtain several thermodynamics properties in the isobaric-isothermal ensemble [8]. This method can be used to calculate derivative properties of inert explosives, but this can not be applied to the detonation products mixture. Indeed, the system after the detonation is under chemical equilibrium, and the derivative properties should be calculated in the appropriate statistical ensemble: the Reaction Ensemble. In fact, although this ensemble has already been used in several conditions (see [4, 5, 7, 9-16] for examples), no derivative properties calculation has already been performed in this ensemble to our knowledge. In this work, we derive the equations to propose a fluctuation formula that allows the calculation of the main derivative properties under chemical equilibrium conditions, performing a single ReMC simulation.

This paper is organized as follow. In a first part, we present the two particular methods used to obtain our results: the ReMC method and the AE-EOS method, and we present a way to establish the fluctuation formula used to calculate derivative properties following the example of Lagache et al. [8]. In a second part, simulation details are presented together with the results obtained for the five systems of interest. Then, discussion and conclusions are provided at the end.

\section{METHODS}

\section{A. AE-EOS Method}

In order to compute the hugoniot curve of a system, we employed the Adaptive Erpenbeck Equation Of State (AE-EOS) method proposed by Brennan and Rice [7]. As discussed in this paper, it is possible to implement this method following several algorithms. We did not follow the idea of Brennan, who chose a succession of
NPT simulations to converge to the Hugoniot pressure at a given temperature. Indeed the hugoniot curve is usually plotted in the (us,up) - shock velocity vs particle velocity - diagram because it corresponds to direct observable of the system. Then, it is possible to plot the Hugoniot in the $(\mathrm{P}, \mathrm{V})$ diagram, using the Hugoniot relations. The temperature is not a direct measure: its calculation is not possible from the Hugoniot relations, and it generally implies the use of particular approximations. In these conditions, temperature does not seem to be the most relevant quantity to use as a constrain. Instead, we proposed here a way to implement the AE-EOS method based on a succession of NVT simulations. In our simulations, the volume is constrained and the temperature is adjusted in order to fulfill the hugoniot conditions. Finally, this method appears more efficient considering that NVT simulations converge more quickly than NPT simulations.

Starting from an initial configuration, the system is simulated in the canonical ensemble $\left(\mathrm{NVT}_{1}\right)$ and the following expression, corresponding to the hugoniot difference $H_{g}(1)$ is evaluated by averaging over several hundred of thousand iterations:

$$
H_{g}=E-E_{0}-\frac{1}{2}\left(P+P_{0}\right)\left(V_{0}-V\right)
$$

where $E_{0}, P_{0}$ and $V_{0}$ are the energy, pressure and volume of the inert explosive before the detonation (the pole of the hugoniot). In fact, this term measures the gap between the simulated thermodynamic state and the real hugoniot state.

After several hundred of thousand iterations (typically 500000 in our case), the temperature of the simulation is slightly modified from $T_{1}$ to $\mathrm{T}_{2}( \pm 10 \mathrm{~K})$. During the following 500000 iterations, $H_{g}(2)$ is evaluated. At the end of this second step, the following derivative is evaluated: 


$$
\frac{d H_{g}}{d T}(2)=\frac{H_{g}(1)-H_{g}(2)}{T_{1}-T_{2}}
$$

From here, the new temperature of the simulation $\mathrm{T}_{3}$ is calculated through:

$$
T_{3}=T_{2}-\frac{H_{g}(2)}{\frac{d H_{g}}{d T}(2)}
$$

and the simulations $\mathrm{NVT}_{3}$ is performed for 500000 iterations, during which $\mathrm{H}_{g}(3)$ is evaluated.

This process is automatically iterated every $5.10^{5}$ steps, evaluating each time:

$$
\frac{d H_{g}}{d T}(n)=\frac{H_{g}(n-1)-H_{g}(n)}{T_{n-1}-T_{n}}
$$

and a new temperature is calculated using:

$$
T_{n+1}=T_{n}-\frac{H_{g}(n)}{\frac{d H_{g}}{d T}(n)}
$$

until the hugoniot difference $\mathrm{H}_{g}(\mathrm{n})$ has converged to the required accuracy. Once the Hugoniot temperature $\mathrm{T}_{\text {hug }}$ is reached, an additional $N V T_{\text {hug }}$ simulation could be performed in order to compute accurately the pressure $\mathrm{P}_{\text {hug. The advantage of this method is its ra- }}$ pidity. The convergence on the hugoniot curve is usually obtained after $5 \cdot 10^{6}$ steps only performed in the NVT ensemble (this corresponds to 10 temperature changes). This remains accurate even when simulations are performed in the Reaction NVT ensemble, where chemical equilibrium is reached within the first 200000 iterations for each NVT simulations.

\section{B. ReMC method}

The goal of the ReMC method is to compute thermodynamic properties of a multi component system at chemical equilibrium. In order to fulfill this condition, a particular statistical ensemble is defined: the Reaction
Ensemble. The complete definition of this ensemble, and the rigourous way to obtain its density probability have been done firstly by Smith and Triska [4]. Here, we will only give a brief description of this ensemble and we invite interested readers to see [4] for more details.

We consider a system of $s$ different chemical species $a_{i}$, and begin by writing the usual canonical ensemble density probability for a non reacting system [17]:

$$
\begin{aligned}
\rho_{\text {ens }}= & \prod_{i=1}^{s} \frac{V^{N_{i}}}{N_{i} ! \Lambda_{i}^{3 N_{i}}} \cdot \\
& \quad \exp \left(-\beta U+\sum_{i=1}^{s} \beta N_{i} \mu_{i}\right) \cdot \mathcal{Q}_{\text {ens }}^{-1}
\end{aligned}
$$

where $N_{i}$ is the number of molecules of species $i, V$ is the volume of the system, $\Lambda_{i}$ is the de Broglie wavelength of species $i$, $\mathrm{U}$ is the configurational energy of the system, and $\mu_{i}$ is the chemical potential of species $i$. $\mathcal{Q}_{e n s}$ is the partition function of the canonical ensemble.

In the Reaction Ensemble, the temperature and the volume (or pressure) are fixed. In comparison with the canonical ensemble, two more constraints are applied to satisfy the chemical equilibrium:

- the number of atoms is fixed for each atom type, so the number of different molecules in the system is controlled by the chemical equation which defines the chemical equilibrium:

$$
\sum_{i=1}^{s} \nu_{i} a_{i}=0
$$

for example:

$$
2 \mathrm{NH}_{3} \rightleftarrows \mathrm{N}_{2}+3 \mathrm{H}_{2}
$$

- the sum of chemical potentials over the different molecule species implied in the 
chemical reaction, weighted by stœechiometric coefficients, is equal to zero:

$$
\sum_{i=1}^{s} \nu_{i} \mu_{i}=0
$$

for exemple:

$$
\mu_{N_{2}}+3 \mu_{H_{2}}=2 \mu_{N H_{3}}
$$

The key point is to establish a Metropolis algorithm that satisfies those constraints over the simulation. To obtain the mechanical equilibrium of the system, the usual Monte Carlo moves can be used: translation, rotation and internal relaxation of molecules. The probability to accept a move is given by [18]:

$$
P_{a c c}=\min \left(1, \exp \left(\frac{\rho_{\text {ens }}(\text { new })}{\rho_{\text {ens }}(\text { old })}\right)\right)
$$

where $\rho_{\text {ens }}\left(\right.$ new) (respectively $\rho_{\text {ens }}($ old $)$ ) is the density probability of the new (respectively old) configuration in the statistical ensemble.

So, following equation 6 , it can be shown that in the case of usual moves, only the total energy of the system varies between the two configurations. We obtain:

$$
P_{a c c}=\min (1, \exp (-\beta \Delta U))
$$

where $\Delta U=U($ new $)-U($ old $)$.

To obtain the chemical equilibrium, we used an additional move: the so-called reaction move, proposed at the same time by Smith and al. [4] and Johnson and al. [5]. This move consists first in choosing a direction to perform the reaction, secondly in deleting a set of reactant molecules randomly chosen in the system, and finally inserting product molecules. Following the example given before, the reaction move can be:

$$
2 \mathrm{NH}_{3} \rightarrow \mathrm{N}_{2}+3 \mathrm{H}_{2}
$$

where two randomly chosen $\mathrm{NH}_{3}$ molecules are deleted and one $N_{2}$ and three $H_{2}$ molecules are inserted, or:

$$
\mathrm{N}_{2}+3 \mathrm{H}_{2} \rightarrow 2 \mathrm{NH}_{3}
$$

where one $\mathrm{N}_{2}$ and three $\mathrm{H}_{2}$ molecules randomly selected are deleted, and two $\mathrm{NH}_{3}$ molecules are inserted.

During this move, not only the energy is modified, but also the respective number of molecules involved in the reaction. Introducing the parameter $\xi$, positive (respectively negative) if the reaction move is performed in the forward (backward) direction, it is possible to show that the ratio between $\rho_{\text {ens }}($ new) and $\rho_{\text {ens }}($ old $)$ is:

$$
\begin{aligned}
\frac{\rho_{\text {ens }}(\text { new })}{\rho_{\text {ens }}(\text { old })}= & \prod_{i=1}^{s} \frac{V^{\xi \nu_{i}} N_{i} !}{\left(N_{i}+\xi \nu_{i}\right) ! \Lambda_{i}^{3 \xi \nu_{i}}} \\
& \quad \exp \left(-\beta \Delta U+\xi \beta \sum_{i=1}^{s} \nu_{i} \mu_{i}\right)
\end{aligned}
$$

Following equation (9), the second term of the exponential should vanish. Nevertheless, as recommended by Ungerer et al. [19], it is important to clarify the definition of the chemical potential in our equations before such simplification.

In equation (9), the chemical potential is the one commonly used in chemistry. The zero of the chemical potential scale (the reference state) in chemistry is usually the chemical potential of elements of the periodic table taken in the standard state (pure element at $P_{0}$ and $\mathrm{T}$ ). In the following, this chemical potential scale is noted $\hat{\mu}$. Equation (9) could then be rewritten:

$$
\sum_{i=1}^{s} \nu_{i} \hat{\mu}_{i}=0
$$

In equations (6) and (15), the chemical potential is the one calculated during the simulation through the Widom test insertion: 


$$
\mu_{i}=-\frac{1}{\beta} \ln \left\langle\frac{V}{\left(N_{i}+1\right) \Lambda_{i}^{3}} \exp \left(-\beta \Delta U^{+}\right)\right\rangle
$$

where $\Delta U^{+}$is the change in energy due to the insertion of a molecule $i$. The zero of this chemical potential scale is obtained for $\mu_{i}=0$, i.e. for a molecule $i$ whose insertion energy is equal to zero in a system of density equals to $\Lambda_{i}^{-3}$. These two scales have a different reference state and the simplification in equation (15) cannot be done straightforwardly. Indeed, it appears necessary to first link these two scales. This is achieved by expressing the chemical potential of a perfect gas in both cases. In the chemistry scale, the chemical potential of a perfect gas is equal to the standard formation free enthalpy of one mole of perfect gas at $P_{0}$ and T:

$$
\hat{\mu}_{i(G P)}=\frac{\Delta_{f} G_{i}^{0}(T)}{\mathcal{N}_{A}}
$$

In the simulation scale, the chemical potential of a perfect gas is equal to:

$$
\mu_{i(G P)}=-\frac{1}{\beta} \ln \left\langle\frac{1}{\beta P_{0} \Lambda_{i}^{3}} \exp \left(-\beta \Delta U_{i n t}^{+}\right)\right\rangle
$$

And we can write:

$$
\mu_{i}-\mu_{i(G P)}=\hat{\mu}_{i}-\hat{\mu}_{i(G P)}
$$

and

$$
\sum_{i=1}^{s} \nu_{i} \mu_{i}=\sum_{i=1}^{s} \nu_{i}\left(\hat{\mu}_{i}-\hat{\mu}_{i(G P)}+\mu_{i(G P)}\right)
$$

This becomes, following equation (9):

$$
\sum_{i=1}^{s} \nu_{i} \mu_{i}=\sum_{i=1}^{s} \nu_{i}\left(\hat{\mu}_{i(G P)}+\mu_{i(G P)}\right)
$$

Finally, introducing $\bar{\nu}=\sum_{i=1}^{s} \nu_{i}$, and using equations (15), (18) and (19), simple algebra leads to the following result:

$$
\begin{aligned}
\frac{\rho_{\text {ens }}(\text { new })}{\rho_{\text {ens }}(\text { old })} & =\left(P_{0} \beta V\right)^{\xi \bar{\nu}} \exp \left(-\xi \frac{\Delta_{r} G^{0}(T)}{R T}\right) . \\
& \prod_{i=1}^{s} \frac{N_{i} !}{\left(N_{i}+\xi \nu_{i}\right) !} \frac{\exp (-\beta \Delta U)}{\left\langle\exp \left(-\beta \Delta U_{\text {int }}^{+}\right)\right\rangle^{\xi \nu_{i}}}
\end{aligned}
$$

In our case, this expression simplifies because we used only molecules represented by a single exponential-6 force centre (i.e. without internal energy contribution):

$$
\begin{aligned}
\frac{\rho_{\text {ens }}(\text { new })}{\rho_{\text {ens }}(\text { old })}= & \left(P_{0} \beta V\right)^{\xi \bar{\nu}} \exp \left(-\xi \frac{\Delta_{r} G^{0}(T)}{R T}\right) . \\
& \prod_{i=1}^{s} \frac{N_{i} !}{\left(N_{i}+\xi \nu_{i}\right) !} \exp \left(-\beta \Delta U_{\text {ext }}\right)
\end{aligned}
$$

Finally, the acceptance probability along a reaction move is given by:

$$
\begin{array}{r}
P_{a c c}=\min \left(1,\left(P_{0} \beta V\right)^{\xi \bar{\nu}} \exp \left(-\xi \frac{\Delta_{r} G^{0}(T)}{R T}\right) .\right. \\
\left.\prod_{i=1}^{s} \frac{N_{i} !}{\left(N_{i}+\xi \nu_{i}\right) !} \exp \left(-\beta \Delta U_{\text {ext }}\right)\right)
\end{array}
$$

Practically, when a reaction move is chosen, the algorithm is the following:

- The direction of the move is randomly chosen $(\xi=0$ or 1$)$. This determines the type $\mathcal{R}$ of reactant molecules to be deleted and $\mathcal{P}$ product molecules to be inserted.

- $\nu_{i}$ molecules of each reactant type $\mathcal{R}$ are randomly chosen and deleted from the configuration.

- $\nu_{j}$ molecules of each product type $\mathcal{P}$ are randomly inserted in the configuration (i.e. the place of insertion is randomly chosen).

- The energy of the new configuration is calculated, and the acceptance probability of the move is obtained using equation (25). 
- According to the Metropolis algorithm, the new configuration is accepted or rejected in the markovian chain depending on the comparison between the value of $P_{a c c}$ with a real randomly taken between 0 and 1.

Combining translation, rotation and reaction moves, it is possible to simulate a chemical equilibrium at a given temperature and density. Nevertheless, the algorithm presented above is limited to low density systems only. Indeed, as density increases, the insertion of product molecules becomes difficult. This limitation should be overcome if one wants to compute hugoniot curves at high density.

The question regarding the insertion of molecule in dense phases is well known, and appears similarly in the Gibbs Ensemble, or in the Grand Canonical ensemble. This problem is usually solved by the use of a statistical bias in the algorithm that enhances the insertion probability. As an example, John Brennan has proposed the use of the cavity bias sampling to improve the insertion of molecules during a reaction move [12]. We rather choose to use the pre-insertion bias [20], already implemented in our MC code, but the two algorithms remain quite similar. This bias applied to a reaction move consists first of all on inserting the first product molecules in the empty space left by the deleted reactant molecules. If more product molecules have to be inserted (i.e. if $\bar{\nu}>0$ ), the insertion is performed at a preselected location using the following criterium: for each insertion, a set of $k$ locations are tested, and one position $x$ is selected following the acceptance criterion:

$$
P(x)=\frac{\exp \left(-\beta U_{L J}(x)\right)}{W}
$$

with $W$, the Rosenbluth factor:

$$
W=\sum_{j=1}^{k} \exp \left(-\beta U_{L J}(j)\right)
$$

In our simulation, this bias has been used for the insertion of the $\mathcal{P}$ molecules of product, with $k=1$ for the $\mathcal{R}$ first molecules of product, inserted at the place released by the $\mathcal{R}$ deleted reactant molecules, and $k=20$ for the $(\mathcal{P}-\mathcal{R})$ last molecules if $\mathcal{P}>\mathcal{R}$.

Two important points have to be considered when using a statistical bias. The first one is that, in order to preserve microreversibility of the Metropolis algorithm, it is necessary to always perform the move in the same way, in the forward or backward direction. More precisely, if the move $A+B \rightarrow C+D+E$ is performed by deleting $A$ and $B$, then replacing $A$ by $C$ and $B$ by $D$ and finally inserting $E$ using the bias, the move $C+D+E \rightarrow A+B$ must be performed by first deleting $C, D$ and $E$, and then by replacing $C$ by $A$ and $D$ by $B$. The second important point is to take into account in the acceptance probability the fact that the move is not random anymore. In fact, a new term should be introduced in the acceptance probability, and for a biased move, we obtain:

$$
P_{a c c}=\min \left(1, \exp \left(\frac{\rho_{\text {ens }}(n e w) P_{g e n}(n \rightarrow o)}{\rho_{\text {ens }}(\text { old }) P_{\text {gen }}(o \rightarrow n)}\right)\right)
$$

where $P_{g e n}(n \rightarrow o)$ (respectively $P_{g e n}(o \rightarrow$ $n)$ ) is the probability to generate the old (new) configuration from the new (old) one using the bias. In the case of the pre-insertion bias applied to our reaction move, we obtain (see [20] for detailed description of the bias):

$$
P_{\text {gen }}(o \rightarrow n)=\prod_{p=1}^{\mathcal{R}} 1 \cdot \prod_{p=\mathcal{R}+1}^{\mathcal{P}} \frac{\exp \left(-\beta U_{L J}\left(x_{p}\right)\right)}{W\left(x_{p}\right)}
$$

where $x_{P}$ are the $\mathcal{P}$ locations where the product molecules are inserted. Note that the second term vanishes if $\mathcal{R} \geqslant \mathcal{P}$.

In the same way:

$$
P_{\text {gen }}(n \rightarrow o)=\prod_{r=1}^{\mathcal{P}} 1 \cdot \prod_{r=\mathcal{P}+1}^{\mathcal{R}} \frac{\exp \left(-\beta U_{L J}\left(x_{r}\right)\right)}{W\left(x_{r}\right)}
$$


where $x_{r}$ are the $\mathcal{R}$ locations where the reactant molecules were placed before the move. Note that the second term vanishes if $\mathcal{P} \geqslant \mathcal{R}$

Finally, if the move is performed in the sens for which $\mathcal{P}>\mathcal{R}$, the acceptance probability is:

$$
\begin{array}{r}
P_{a c c}=\min \left(1,\left(P_{0} \beta V\right)^{\xi \bar{\nu}} \exp \left(-\xi \frac{\Delta_{r} G^{0}(T)}{R T}\right) .\right. \\
\prod_{i=1}^{s} \frac{N_{i} !}{\left(N_{i}+\xi \nu_{i}\right) !} \exp \left(-\beta \Delta U_{e x t}\right) . \\
\left.\prod_{p=\mathcal{R}+1}^{\mathcal{P}} \frac{\exp \left(-\beta U_{L J}\left(x_{p}\right)\right)}{W\left(x_{p}\right)}\right)
\end{array}
$$

And, if the move is performed in the sens for which $\mathcal{R}>\mathcal{P}$, the acceptance probability is:

$$
\begin{array}{r}
P_{a c c}=\min \left(1,\left(P_{0} \beta V\right)^{\xi \bar{\nu}} \exp \left(-\xi \frac{\Delta_{r} G^{0}(T)}{R T}\right) .\right. \\
\prod_{i=1}^{s} \frac{N_{i} !}{\left(N_{i}+\xi \nu_{i}\right) !} \exp \left(-\beta \Delta U_{e x t}\right) . \\
\left.\prod_{r=\mathcal{P}+1}^{\mathcal{R}} \frac{\exp \left(-\beta U_{L J}\left(x_{r}\right)\right)}{W\left(x_{r}\right)}\right)
\end{array}
$$

It is interesting to note that the only input data needed is the $\Delta_{r} G^{0}(T)$ of the simulated reaction. This standard free enthalpy of reaction is easily obtained from experimental database.

It is also possible to compute a chemical equilibrium combining several chemical reactions. To do this, we used the same algorithm as presented above, but we added a preliminary step consisting on randomly choosing the reaction to perform before each reaction move.

Finally, note that it is also possible to simulate a chemical equilibrium at constant pressure. To do this, an other move has to be used during the simulation: the usual volume change Monte Carlo movement. As in the NPT ensemble, it consists on modifying the volume of the simulation box, performing an homothetic transformation of the system. The acceptance probability of this move is given by [17]:

$$
\begin{aligned}
P_{\text {acc }}=\min [1, \exp ( & -\beta(\Delta U+P \Delta V \\
& \left.\left.\left.+N \ln \left(\frac{V_{\text {new }}}{V_{\text {old }}}\right)\right)\right)\right]
\end{aligned}
$$

\section{Fluctuation Formula in the Reaction Ensemble}

In this section, starting with the Reaction Ensemble partition function defined by Smith [4], we will develop equations following the example of Lagache et al. [8] in order to establish the fluctuation formula for thermodynamic derivative properties. We will use capital letters $X$ for extensive properties, and lower case letters $x$ for the associated molar properties.

As discussed previously, the system is composed of $s$ molecular species assuming the following chemical equilibrium:

$$
\sum_{i=1}^{s} \nu_{i} \mu_{i}=0
$$

where $\mu_{i}$ is the chemical equilibrium of molecular type $i$ and $\nu_{i}$ is the stochiometric coefficient of $i$. This condition can be considered as an external constrain imposed on the thermodynamic system. As a consequence, Smith and Triska have established the expression of the Reaction Ensemble partition function under chemical equilibrium from a Legendre transformation [4]:

$$
\begin{aligned}
Q= & \sum_{\xi} \prod_{i=1}^{s} \frac{\left(V(\xi) q_{i}\right)^{N_{i}(\xi)}}{\Lambda_{i}^{3 N_{i}(\xi)} N_{i}(\xi) !} \\
& \times \int \exp \left[-\beta \hat{H}\left(z_{1}, \ldots\right)\right] d z_{1} \ldots
\end{aligned}
$$

where $\xi$ is the extent of the chemical reaction used to define all the accessible states 
of the system: for a given molecular type $i$, $N_{i}(\xi)=N_{i}^{\circ}+\xi \nu_{i}$, with $N_{i}(\xi)$ the number of molecules of type $i$ for a given state, and $N_{i}^{\circ}$ the initial number of molecules of type $i$. In equation (35), $V(\xi)$ is the volume of the simulation box, $q_{i}$ is the part of the partition function corresponding to an isolated molecule of type $i, \Lambda_{i}$ is the de Broglie thermal wavelength, and $\hat{H}$ is the configurational enthalpy, $\hat{H}=U^{e x t}+U^{i n t}+P V$, expressed in terms of the relative coordinates $z_{i}$ of the molecules.

As done in previous parts, we set:

$$
\frac{q_{i}}{\Lambda_{i}^{3}}=P_{0} \beta \times \exp \left[-\beta \frac{\Delta_{f} G_{i}{ }^{\circ}(T)}{\mathcal{N}_{\mathcal{A}}}\right]
$$

As a consequence, the Reaction Ensemble partition function can be written:

$$
Q=\sum_{\xi} \prod_{i=1}^{s} \frac{\left(P_{0} \beta V(\xi)\right)^{N_{i}(\xi)}}{N_{i}(\xi) !} \times \exp \left[-\beta N_{i}(\xi) \frac{\Delta_{f} G_{i}^{\circ}(T)}{\mathcal{N}_{\mathcal{A}}}\right] \times \int \exp \left[-\beta \hat{H}\left(z_{1}, \ldots\right)\right] d z_{1} \ldots
$$

Then, an average property $X$ in this ensemble is given by:

$$
\langle X\rangle=Q^{-1} \sum_{\xi} \prod_{i=1}^{s} \frac{\left(P_{0} \beta V(\xi)\right)^{N_{i}(\xi)}}{N_{i}(\xi) !} \times \exp \left[-\beta N_{i}(\xi) \frac{\Delta_{f} G_{i}{ }^{\circ}(T)}{\mathcal{N}_{\mathcal{A}}}\right] \times \int X \exp \left[-\beta \hat{H}\left(z_{1}, \ldots\right)\right] d z_{1} \ldots
$$

To obtain the expression of calorific capacity, it is necessary to derive this expression with respect to temperature. Two quantities depend directly on $\mathrm{T}$ in equation (38): $\beta$ and $\Delta_{f} G_{i}{ }^{\circ}(T)$. Thus, through simple algebra and using properties of perfect gas concerning $\Delta_{f} G_{i}{ }^{\circ}(T)$, it can be shown that:

$$
\begin{aligned}
\left(\frac{\partial\langle X\rangle}{\partial \beta}\right)_{P}= & (\langle X\rangle\langle\hat{H}\rangle-\langle X \hat{H}\rangle)-\frac{1}{\beta}\left(\langle X\rangle\left\langle N_{t o t}\right\rangle-\left\langle X N_{t o t}\right\rangle\right)+ \\
& \left(\langle X\rangle\left\langle\sum_{i=1}^{s} \frac{N_{i}}{\mathcal{N}_{\mathcal{A}}} \Delta_{f} H_{i}{ }^{\circ}(T)\right\rangle-\left\langle X \sum_{i=1}^{s} \frac{N_{i}}{\mathcal{N}_{\mathcal{A}}} \Delta_{f} H_{i}{ }^{\circ}(T)\right\rangle\right)
\end{aligned}
$$

where $N_{t o t}=\sum_{i=1}^{s} N_{i}$ is the total number of molecules which can fluctuate during the simulation, and $\Delta_{f} H_{i}{ }^{\circ}(T)$ is the standard entalpy of formation of molecular type $i$ at temperature $\mathrm{T}$.

In fact, in comparison with the fluctuation formula obtained in the isobare-isotherm ensemble [8], the equation (39) takes into account not only the fluctuations of energy, but also the fluctuations of composition and number of molecules. From this equation, taking $X=V$, the coefficient of thermal expansion $\alpha_{P}$ can be calculated:

$$
\alpha_{P}=\frac{1}{\langle V\rangle}\left(\frac{\partial\langle V\rangle}{\partial T}\right)_{P}
$$

The derivation of calorific capacity is a little more complicated. Indeed, it needs the derivation of total energy in regard with temperature, including kinetic energy. But in a Monte Carlo simulation, the kinetic energy $K$ is not taken into account, and as a consequence, only the residual calorific capacity can be calculated during the simulation. Thus, Lagache et al. 
have proposed to clearly separate the two contributions, ideal and residual:

$$
C_{P}(T, P)=C_{P}^{i d}(T)+C_{P}^{r e s}(T, P)
$$

with

$$
C_{P}^{i d}(T)=\left(\frac{\partial\left\langle H^{i d}\right\rangle}{\partial T}\right)_{P}
$$

and

$$
C_{P}^{r e s}(T, P)=\left(\frac{\partial\left\langle H^{r e s}\right\rangle}{\partial T}\right)_{P}
$$

Considering that $H^{i d}$ can not be evaluated in regard with $K$, but that it is possible to give an expression of it in regard with standard thermodynamical data $\left(H^{i d}=\sum_{i}\left\langle N_{i}\right\rangle \frac{\Delta_{f} H_{i}^{\circ}}{\mathcal{N}_{\mathcal{A}}}\right)$, we obtain:

$$
C_{P}^{i d}(T)=\sum_{i} \frac{\partial\left\langle N_{i}\right\rangle}{\partial T} \frac{\Delta_{f} H_{i}^{\circ}}{\mathcal{N}_{\mathcal{A}}}+\sum_{i}\left\langle N_{i}\right\rangle C_{P_{i}}^{\circ}
$$

and with $H^{\text {res }}=U^{e x t}+P V-N_{t o t} k T$, we can thus obtain $C_{P}^{\text {res }}(T, P)$, and then $C_{P}(T, P)$, using equation (39) with $X=H^{\text {res }}$ and $X=N_{i}$.

To obtain the other derivative properties of interest, it is necessary to calculate the isothermal compressibility $\beta_{T}$ whose expression is the same as in the isothermal-isobaric ensemble:

$$
\begin{aligned}
\beta_{T} & =-\frac{1}{\langle V\rangle}\left(\frac{\partial\langle V\rangle}{\partial P}\right)_{T} \\
& =\frac{1}{\langle V\rangle k T}\left(\left\langle V^{2}\right\rangle-\langle V\rangle^{2}\right)
\end{aligned}
$$

Then it is possible to obtain the calorific capacity at constant volume $C_{V}$, the sound velocity $C_{S}$ and the Grüneisen coefficient $\gamma$ :

$$
\begin{aligned}
& C_{V}=C_{P}-\frac{\alpha_{P}^{2} V T}{\beta_{T}} \\
& C_{S}=\sqrt{\frac{V C_{P}}{C_{V} \beta_{T}}} \\
& \gamma=\frac{V \alpha_{P}}{C_{v} \beta_{T}}
\end{aligned}
$$

It is important to note that the quantity $X$ (for example $C_{P}(T, P)$ ) is extensive. To obtain the exact molar quantity, it is necessary to derive the molar quantity of $X$ (for example the molar enthalpies $h^{i d}$ and $h^{r e s}$ ), introducing again the $N_{i}$ 's in the derivative, for which fluctuations have to be taken into account. Nevertheless, the only constant quantity during a detonation is the mass of the system, and the use is to employ massic quantities, so we did not evaluate molar quantity in this work.

\section{RESULTS}

\section{A. Simulation Details}

All the simulations performed in this work have been done with the program GIBBS, owned by the Institut Francais du Petrole, the Université Paris Sud, and the CNRS, and developped in collaboration between those three owners and the CEA [19].

In order to reach the chemical equilibrium of a system using the ReMC method, it is necessary to define a priori the set of chemical equations driving the system. To do this, the first step is to choose which type of molecule is expected at equilibrium. In our case, it is known that the detonation products of NiMe, HNE, TNM, PETN and RDX are the following: $\mathrm{CO}_{2}, \mathrm{H}_{2} \mathrm{O}, \mathrm{CO}, \mathrm{N}_{2}, \mathrm{H}_{2}, \mathrm{O}_{2}, \mathrm{NO}$, $\mathrm{NH}_{3}$ and $\mathrm{CH}_{4}$ in a fluid phase, and eventually carbon in a solid phase. More precisely, it is possible to neglect some of those molecules, because their molar fractions are known to be very small. So on, table I shows the detonation products taken into account for each studied explosives.

The second step consists on finding a set of linearly independent chemical equations involving those molecules that satisfy the stœechiometry and the chemical specificity of the system. To do this, we applied the formula-vector matrix stochiometric algorithm devised by Smith 


\begin{tabular}{|c|c|c|c|c|c|}
\hline & NiMe & TNM & HNE & PETN & RDX \\
\hline \hline $\mathrm{CO}_{2}$ & $\mathrm{x}$ & $\mathrm{x}$ & $\mathrm{x}$ & $\mathrm{x}$ & $\mathrm{x}$ \\
$\mathrm{H}_{2} O$ & $\mathrm{x}$ & & & $\mathrm{x}$ & $\mathrm{x}$ \\
$\mathrm{CO}$ & $\mathrm{x}$ & & $\mathrm{x}$ & $\mathrm{x}$ & $\mathrm{x}$ \\
$N_{2}$ & $\mathrm{x}$ & $\mathrm{x}$ & $\mathrm{x}$ & $\mathrm{x}$ & $\mathrm{x}$ \\
$\mathrm{H}_{2}$ & $\mathrm{x}$ & & & $\mathrm{x}$ & $\mathrm{x}$ \\
$\mathrm{O}_{2}$ & & $\mathrm{x}$ & $\mathrm{x}$ & & \\
$N O$ & & $\mathrm{x}$ & $\mathrm{x}$ & $\mathrm{x}$ & $\mathrm{x}$ \\
$\mathrm{NH}_{3}$ & $\mathrm{x}$ & & & $\mathrm{x}$ & $\mathrm{x}$ \\
$\mathrm{CH}_{4}$ & $\mathrm{x}$ & & & & \\
$C$ solid & $\mathrm{x}$ & & & & \\
\hline
\end{tabular}

TABLE I: Molecular species taken into account in the detonation products mixture of the studied explosives.

and Missen [21]. It is important to note that the nitromethane case is particular due to the presence of solid carbon. We will show later that the solid phase is not included in the chemical equilibrium, and this is the reason why it has not been included in the formula-vector matrix stoechiometric algorithm used here. This method leads to the following set of reactions:

- NiMe:

$$
\begin{aligned}
& 2 \mathrm{NH}_{3} \rightleftarrows \mathrm{N}_{2}+3 \mathrm{H}_{2} \\
& \mathrm{CO}+2 \mathrm{NH}_{3} \rightleftarrows \mathrm{N}_{2}+\mathrm{CH}_{4}+\mathrm{H}_{2} \mathrm{O} \\
& \mathrm{CO}_{2}+\mathrm{H}_{2} \rightleftarrows \mathrm{CO}+\mathrm{H}_{2} \mathrm{O}
\end{aligned}
$$

- TNM (All the carbon atoms are in the $\mathrm{CO}_{2}$ molecules):

$$
2 \mathrm{NO} \rightleftarrows \mathrm{O}_{2}+\mathrm{N}_{2}
$$

- HNE:

$$
\begin{gathered}
2 \mathrm{NO} \rightleftarrows \mathrm{O}_{2}+\mathrm{N}_{2} \\
2 \mathrm{CO}_{2} \rightleftarrows 2 \mathrm{CO}+\mathrm{O}_{2}
\end{gathered}
$$

- PETN and RDX:

$$
\begin{aligned}
2 \mathrm{NH}_{3} & \rightleftarrows \mathrm{N}_{2}+3 \mathrm{H}_{2} \\
2 \mathrm{CO}_{2}+\mathrm{N}_{2} & \rightleftarrows 2 \mathrm{CO}+2 \mathrm{NO} \\
\mathrm{CO}_{2}+\mathrm{H}_{2} & \rightleftarrows \mathrm{CO}+\mathrm{H}_{2} \mathrm{O}
\end{aligned}
$$

The potential models used in this study are the exponential- 6 potential proposed by Fried et al. [22] that reproduce the thermodynamic behavior of pure compounds under high temperatures and pressures. A cut-off equal to the half of the box length has been used to reduce the computing time. Periodic boundary conditions together with long range corrections have been used.

All the simulations performed in this work were initiated with a number of molecules corresponding to a system of several hundred of explosive molecules. During ReMC NVT simulations, the respective probabilities of choosing a translation move and a reaction move were 0.8 and 0.2. During ReMC NPT simulations, the respective probabilities of choosing a translation move, a volume change and a reaction move were $0.8,0.02$ and 0.18 . The pole conditions used to compute $H_{g}$ in the AEEOS method are given in table II. The convergence of AE-EOS ReMC simulations were generally obtained after $10^{7}$ iterations, changing the temperature every $0.5 \cdot 10^{6}$ iterations and calculating the $H_{g}$ value on $0.25 \cdot 10^{6}$ iterations before evaluating the new temperature. The NPT simulations performed to obtain derivative properties should be much longer: around $20 \cdot 10^{6}$ iterations to converge, and averages were calculated on $30 \cdot 10^{6}$ iterations after convergence.

\begin{tabular}{|c|c|c|}
\hline & $V_{0}\left(\mathrm{~cm}^{3} \cdot \mathrm{g}^{-1}\right)$ & $E_{0}\left(\mathrm{~J}^{\mathrm{kg}}{ }^{-1}\right)$ \\
\hline \hline NiMe & 0.883 & $-1.939 \cdot 10^{6}$ \\
TNM & 0.606 & $1.879 \cdot 10^{5}$ \\
HNE & 1.096 & $3.987 \cdot 10^{6}$ \\
PETN & 0.971 & $-1.68 \cdot 10^{6}$ \\
RDX & 1.0 & $3.181 \cdot 10^{5}$ \\
\hline
\end{tabular}

TABLE II: Pole conditions used to compute $H_{g}$ in the AE-EOS method. 


\section{B. Crussard curves}

To calculate the Crussard curves of the four systems without solid phase of carbon (TNM, HNE, PETN, RDX), we have directly applied the AE-EOS ReMC method described previously. Five simulations have been performed for each system, at five different specific volumes between 0.4 and $0.95 \mathrm{~cm} . \mathrm{g}^{-1}$. Monte Carlo results are displayed in figure 1 (TNM), 2 (HNE), 3 (PETN) and 4 (RDX), and compared with results obtained with our thermochemical code using the same potential models [2].

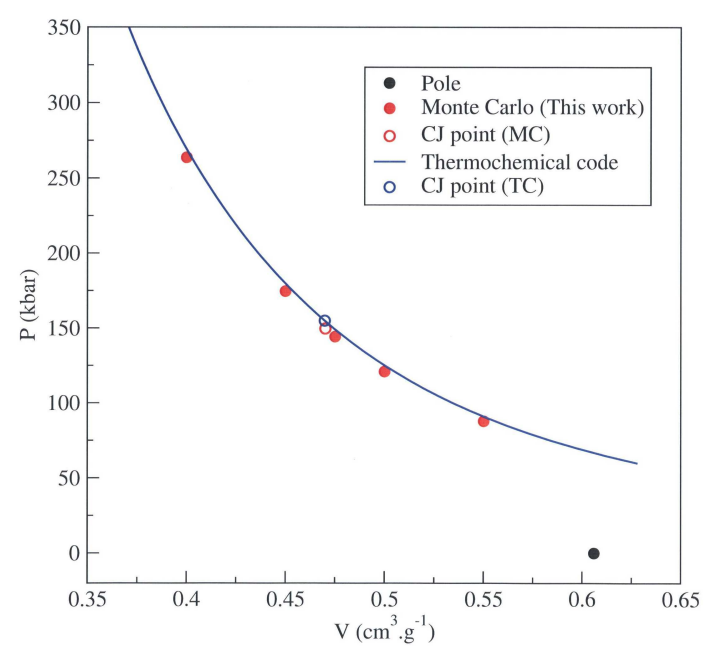

FIG. 1: Calculated Crussard Curve of Tetranitromethane (TNM).

For those four systems, we can see that Monte Carlo results are in good agreement with thermochemical ones. The small discrepancies seen in figures $1,2,3$ and 4 , are probably due to the various approximations used in the thermochemical code, for example in the MCRSR theory [1] and the one-fluid hypothesis. Those results, obtained with the same potential models in both cases, validate our microscopic method to calculate Crussard curves.

Concerning Nitromethane, the simulation process is a little more complicated due to the fact that we are not actually able to explic-

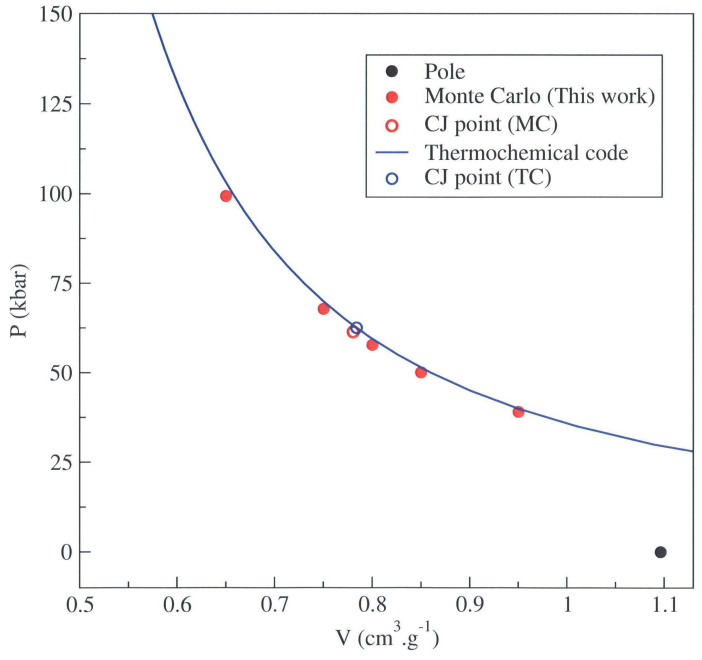

FIG. 2: Calculated Crussard Curve of Hexanitroethane (HNE).

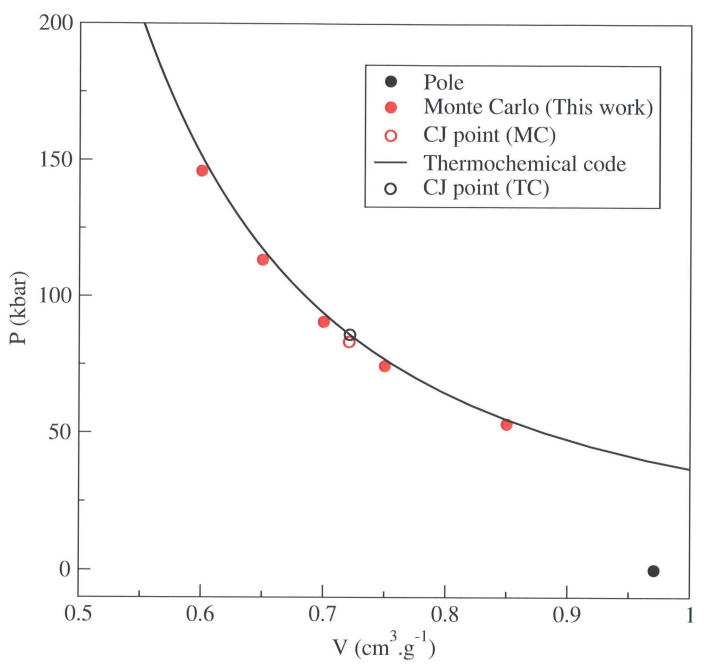

FIG. 3: Calculated Crussard Curve of PETN.

itly simulate a chemical equilibrium involving a solid phase. To obtain the Crussard curve of nitromethane, we then applied the following two steps process:

- In a first step, we performed a calculation of the Crussard curve with our thermochemical code, in order to determine the quantity, the molar volume and the molar energy of the solid phase of carbon along the Crussard curve, using the Reevan Thiel equation of state [23]. 


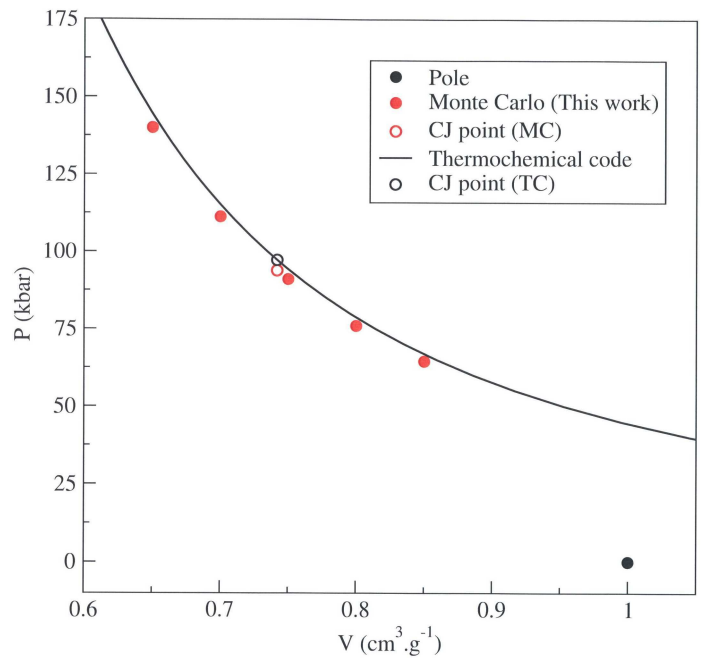

FIG. 4: Calculated Crussard Curve of RDX.

- Then, we performed the five AE-EOS ReMC simulations at five different volumes, but with the stoechiometry corresponding to nitromethane minus the carbon trapped into the solid phase. During those simulations, the AE-EOS method is applied with a modified RankineHugoniot relation:

$$
H_{g}=E_{t o t}-E_{0}-\frac{1}{2}\left(P+P_{0}\right)\left(V_{0}-V_{t o t}\right)
$$

with

$$
\begin{gathered}
E_{\text {tot }}=E_{\text {fluid }}+E_{\text {solid }} \\
V_{\text {tot }}=V_{\text {fluid }}+V_{\text {solid }}
\end{gathered}
$$

where $E_{\text {fluid }}$ and $V_{\text {fluid }}$ are the energy and volume of the fluid calculated during the Monte Carlo simulation, whereas $E_{\text {solid }}$ and $V_{\text {solid }}$ are the energy and volume of the solid phase of carbon taken from the thermochemical calculation.

Results obtained concerning the nitromethane appear in figure 5 .

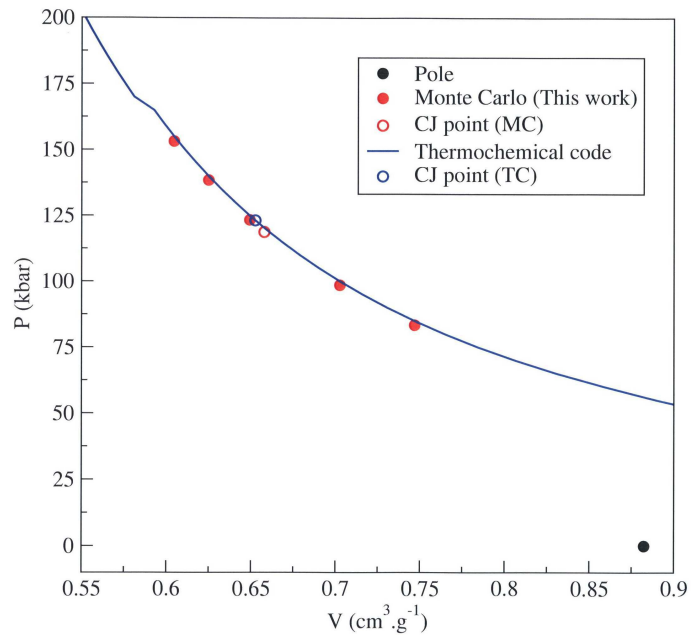

FIG. 5: Calculated Crussard Curve of Nitromethane (NiMe).

As far as nitromethane is concerned, Monte Carlo and thermochemical results are also consistent. Nevertheless, in this case, Monte Carlo results depend on the thermochemical ones through the Ree-van Thiel equation of State for solid phase of carbon. This is not satisfactory, because this means that the accuracy of a complex microscopic Monte Carlo simulation is bound to the quality of an empirical equation of state. Moreover, in our simulations, the quantity of solid carbon is determined a priori.

As a consequence, although we propose in this work a simple and successful way to take into account the solid phase in the fluid chemical equilibrium, this may be improved to better simulate the equilibrium between the solid and fluid phases. Some perspectives of this work are presented in the conclusion.

\section{Chapman-Jouguet State}

Once the Crussard curve has been calculated for each system, the five CJ points could be determined. To do this, we have fitted the calculated points by the following function, which gives the pressure on the Crussard curve in re- 
gard with $\eta=V / V_{0}$ :

$$
P(\eta)=a \cdot e^{-b \eta}+c \cdot \eta^{-d}
$$

The CJ point is then the tangential point between the Rayleigh line and the Crussard curve. Thus, knowing the pole conditions, we are able to give a value of the derivative of the pressure along the Crussard curve with respect to the volume at the CJ point:

$$
\frac{d P(\eta)}{d V}(C J)=\frac{P\left(\eta_{C J}\right)-P_{0}}{V\left(\eta_{C J}\right)-V_{0}}
$$

which is equal to:

$$
\frac{d P(\eta)}{d \eta}(C J)=\frac{P\left(\eta_{C J}\right)-P_{0}}{\eta_{C J}-1}
$$

Introducing equation (50), and assuming that $P_{0}<<P\left(\eta_{C J}\right)$, we obtain the CJ point where $\eta$ satisfies:

$$
\frac{a \cdot e^{-b \eta}+c \cdot \eta^{-d}}{(1-\eta)}=a \cdot b \cdot e^{-b \eta}+d \cdot c \cdot \eta^{-(d+1)}
$$

In this method, the CJ points calculated for the five systems are presented in table III. From the calculated $\mathrm{P}_{C J}$ and $\mathrm{V}_{C J}$, we have determined the detonation velocity $\mathrm{D}_{C J}$, using the following equation derived from the Hugoniot relations:

$$
D_{C J}=V_{0} \sqrt{\frac{\left(P_{C J}-P_{0}\right)}{\left(V_{0}-V_{C J}\right)}}
$$

This property is interesting because it can directly be measured experimentally. Table III shows a comparison between the calculated and measured values of $\mathrm{D}_{C J}$.

Table III shows that the CJ states calculated using our Monte Carlo method are in good

\begin{tabular}{|c|c|c|c|c|c|}
\hline & NiMe & TNM & HNE & PETN & RDX \\
\hline \multicolumn{6}{|c|}{ Monte Carlo Results } \\
\hline $\mathrm{P}_{C J}(\mathrm{kbar})$ & 118.7 & 149.7 & 61.42 & 83.17 & 93.83 \\
\hline $\mathrm{V}_{C J}\left(\mathrm{~cm}^{3} \cdot \mathrm{g}^{-1}\right)$ & 0.658 & 0.470 & 0.780 & 0.721 & 0.742 \\
\hline $\mathrm{T}_{C J}(\mathrm{~K})$ & 3463 & 2250 & 6017 & 4677 & 4325 \\
\hline $\mathrm{D}_{C J}\left(\mathrm{~m} \cdot \mathrm{s}^{-1}\right)$ & 6414 & 6364 & 4828 & 5599 & 6028 \\
\hline \multicolumn{6}{|c|}{ Thermochemical Results } \\
\hline $\mathrm{P}_{C J}(\mathrm{kbar})$ & 123.1 & 155.0 & 62.57 & 85.85 & 97.22 \\
\hline $\mathrm{V}_{C J}\left(\mathrm{~cm}^{3} \cdot \mathrm{g}^{-1}\right)$ & 0.653 & 0.470 & 0.784 & 0.721 & 0.742 \\
\hline $\mathrm{T}_{C J}(\mathrm{~K})$ & 3513 & 2229 & 6042 & 4685 & 4350 \\
\hline $\mathrm{D}_{C J}\left(\mathrm{~m} \cdot \mathrm{s}^{-1}\right)$ & 6493 & 6464 & 4897 & 5696 & 6137 \\
\hline \multicolumn{6}{|c|}{ Experimental Results $^{a}$} \\
\hline $\mathrm{D}_{C J}\left(\mathrm{~m} \cdot \mathrm{s}^{-1}\right)$ & 6370 & 6450 & 4950 & 5620 & 6050 \\
\hline $\begin{array}{c}{ }^{a} \text { Reference }[24] \\
\text { Reference }[25] \\
\text { Reference }[26] \\
\text { Reference }[27] \\
\text { Reference }[28]\end{array}$ & $\begin{array}{l}\text { or NiM } \\
\text { for TNN } \\
\text { for HNP } \\
\text { for PET } \\
\text { for RDY }\end{array}$ & & & & \\
\hline
\end{tabular}
agreement with thermochemical results. The discrepancies between the two methods are below $4 \%$ concerning pressures, below $1 \%$ concerning volumes, below $1.5 \%$ concerning
TABLE III: CJ states of the studied explosives calculated using our Monte Carlo method, using our thermochemical code [2], and, considering the detonation velocity, compared with experimental measurements [24-28].

temperatures and below $1.8 \%$ concerning detonation velocities.

We can also note that the calculated values of $\mathrm{D}_{C J}$ are in good agreement with the experimental values in each of the five cases. The difference between Monte Carlo simulation results and experimental ones is about $1.8 \%$ for nitromethane, $1.6 \%$ for TNM, $1.3 \%$ fo $\mathrm{HNE}$, $0.4 \%$ for PETN and $1.2 \%$ for RDX, which is very satisfactory considering that simple potential models have been used, and considering that experimental uncertainties concerning the positions of the CJ state are in the same order of magnitude. It is already anticipated that MC results would be more significant as realistic potential models become used. It is also important to notice that, considering detonation velocity, thermochemical results are sometimes closer to experiment than Monte Carlo ones. This can be explained by the fact that po- 
tential models are effective potentials. Indeed, they have been optimized through the use of thermochemical codes, and thus integrate the different sources of errors coming from the different approximations.

\section{Derivative properties at the CJ point}

Four ReMC NPT simulations have been performed in order to calculate the derivative properties of TNM, HNE, PETN and RDX. Because of the contribution of the carbon solid state in the CJ point of nitromethane, this system has not been considered. Results are presented in table IV.

First of all, it is important to note that the converged volume obtained at the end of those NPT simulations is consistent with the predicted CJ volume presented in table III.

\begin{tabular}{|c|c|c|c|c|}
\hline & TNM & HNE & PETN & RDX \\
\hline \hline \multicolumn{2}{|c|}{ Monte Carlo Results } \\
\hline $\mathrm{C}_{P}\left({\left.\mathrm{~J} . \mathrm{kg}^{-1} . \mathrm{K}^{-1}\right)}^{-1660}\right.$ & 1826 & 2199 & 2024 \\
$\mathrm{C}_{V}\left({\left.\mathrm{~J} . \mathrm{kg}^{-1} . \mathrm{K}^{-1}\right)} 1568\right.$ & 1593 & 1977 & 1800 \\
$\mathrm{C}_{S}\left(\mathrm{~m} . \mathrm{s}^{-1}\right)$ & 4927 & 3434 & 4177 & 4494 \\
$\Gamma$ & 0.619 & 0.396 & 0.436 & 0.536 \\
\hline \multicolumn{5}{|c|}{ Thermochemical Results } \\
\hline $\mathrm{C}_{P}\left(\mathrm{~J} . \mathrm{kg}^{-1} . \mathrm{K}^{-1}\right)$ & 1660 & 1824 & 2233 & 2085 \\
$\mathrm{C}_{V}\left(\mathrm{~J} . \mathrm{kg}^{-1} . \mathrm{K}^{-1}\right)$ & 1575 & 1592 & 1998 & 1827 \\
$\mathrm{C}_{S}\left({\left.\mathrm{~m} . \mathrm{s}^{-1}\right)}_{\Gamma}\right.$ & 5008 & 3492 & 4233 & 4551 \\
$\Gamma$ & 0.59 & 0.40 & 0.45 & 0.57 \\
\hline
\end{tabular}

TABLE IV: Derivative properties (heat capacities, sound velocities and Grüneisen coefficients) at CJ point for the four studied explosives.

Table IV shows that the derivative properties calculated at the CJ points using our Monte Carlo method are in good agreement with thermochemical results. The discrepancies between the two methods are below $3 \%$ concerning heat capacity at constant pressure $\left(C_{P}\right)$, below $1.5 \%$ concerning heat capacity at constant volume $\left(C_{V}\right)$, below $1.8 \%$ concerning sound velocity $\left(C_{S}\right)$ and around $5 \%$ concerning
Grüneisen coefficient $(\Gamma)$. It is important to note that a part of those discrepancies is due to the fact that the $\left(P_{C J}, T_{C J}\right)$ are not exactly the same.

The calculation of the sound velocity at the predicted CJ state appears really interesting because it allows to verify that the considered thermodynamic state is really the CJ state. Indeed, the CJ state is the only point in the Crussard curve where the following relation is verified: $D_{C J}=C_{S}+u_{P}(C J) . u_{P}$ is the particle velocity, obtained from the Hugoniot relation: $u_{P}=\frac{P-P_{0}}{\rho_{0} D}[29]$. Using the Monte Carlo results presented in tables III and IV, it appears that this relation is verified for the 4 systems, with an accuracy of $0.5 \%$. This is very satisfactory because it validates in the same time the way to determine the CJ state from the Crussard curve, and the fluctuation formula used to calculate $C_{S}$.

Those results concerning derivative properties are important because to our known, it represents the first results of microscopic calculations of such properties for systems under chemical equilibrium.

\section{CONCLUSION}

We have proposed in this work a new microscopic method to calculate the crussard curve and the CJ state of detonation products mixtures of usual explosives. This method, based on Monte Carlo simulations employing ReMC and AE-EOS methods, has been validated by comparison with thermochemical calculations. The simulations presented here have been performed with simple potential models, and it can be expected that simulations performed with complex potentials would give more accurate results. This is the first perspective of our work.

We also have proposed a fluctuation formula to calculate derivative properties in the 
Reaction statistical ensemble. This formula takes into account the energy fluctuations, and the fluctuations of composition and number of molecules. This formula has allowed us to calculate heat capacities, sound velocities and Grüneisen coefficients through ReMC NPT simulations, in the 4 cases where no carbon phase was included. Concerning the last case (NiMe), the calculation of derivative properties was not possible with this formula, because of the way the solid phase has been taken into account.

Finally, we have proposed a simple way to take into account the solid phase of carbon which could appear in the detonation products. In this work, the properties of the solid phase are determined by thermochemical calculations via the Ree-van Thiel equation of state, and included in the Monte Carlo simulation. This leads to a satisfactory restitution of the Crussard curve and the CJ properties, but still presents unconsistency in the treatment of the equilibrium between the fluid mixture and the solid phase. This constitutes the second perspective of this work. We could think about several ways to improve this equilibrium. A first idea should be to explicitly treat the car- bon phase in the simulation. Nevertheless, this would strongly increase the computing time. Moreover, the intrinsic properties of the solid phase are not the main goal of this type of study. So, it should be sufficient to take into account the solid phase in a more implicit way. For example, considering that experimentally, the solid phase of carbon seems to appear as agregates, it could be interesting to simulate those agregates via mesoparticules with more or less interactions with the fluid mixture. Moreover, those improvements should allow us to calculate the derivative properties of system under a chemical equilibrium including a solid phase.

\section{ACKNOWLEDGEMENTS}

Pr P. Ungerer from IFP is gratefully acknowledged for fruitful discussions about the theory of the Reaction Ensemble and the way to obtain the acceptance probability. E. Auroux from CEA is gratefully acknowledged for his help on thermochemical calculations and for his knowledge about experimental data. All Monte Carlo simulations have been performed with the Gibbs code from IFP, CNRS and the Université Paris-Sud [19].
[1] M. Ross. J. Chem. Phys., 71:1567, 1979.

[2] F. Charlet, M.-L. Turkel, J.-F. Danel, and L. Kazmadjian. J. Applied Phys., 84:4227, 1998.

[3] F.H. ReE. J. Chem. Phys., 64:4601, 1763.

[4] W.R. Smith and B. Triska. J. Chem. Phys., 100:3019, 1994.

[5] J.K. Johnson, A.Z. PAnagiotopoulos, and K.E. Gubbins. Mol. Phys., 81(3):717, 1994.

[6] J.J. Erpenbeck. Phys. Rev. A, 46:6406, 1992.

[7] J.K. Brennan and B.M. Rice. Mol. Phys., 101:3309, 2003.

[8] M. Lagache, P. Ungerer, A. Boutin, and A.H. Fuchs. Phys. Chem. Chem. Phys., 3:4333-4339, 2001.

[9] M. Lísal, I. Nezbeda, and W. R. Smith. J. Chem. Phys., 110(17):8597, 1999.
[10] C. H. Tuner, J. K. Johnson, and K. E. GubBIns. J. Chem. Phys., 114(4):1851, 2001.

[11] M. Borówko and R. Zagórski. J. Chem. Phys., 114(12):5397, 2001.

[12] J.K. Brennan. Mol. Phys., 103:2647, 2005.

[13] M. Lísal, M. Bendová, and W. R. Smith. Fluid Ph. Eq., 235:50, 2005.

[14] X. Peng, W. Wang, and S. Huang. Fluid Ph. Eq., 231:138, 2005.

[15] N. Hansen, S. Jakobtorweinen, and F. J. KeIL. J. Chem. Phys., 122:164705, 2005.

[16] J. Carrero-Mantilla and M. LlanoRestrepo. Fluid Ph. Eq., 242:189, 2006.

[17] D. MCQuARRIE. Statistical Mechanics. Harper and Row, 1976.

[18] D. Frenkel and B. Smit. Understanding Molecular Simulation - From Algorithms to 
Applications. Academic Press, 2002.

[19] P. Ungerer, A. Boutin, and B. Tavitian. Applications of Molecular Simulation in the Oil and Gas Industry. IFP Publications, 2005.

[20] E. Bourasseau, P. Ungerer, and A. Boutin. J. Phys. Chem. B, 106:5483, 2002.

[21] W.R. Smith and R.W. Missen. Chem. Eng. Educ., 13:26-32, 1979.

[22] L.E. Fried, W.M. Howard, and P.C. Souers. In proceedings of the 12th International Detonation Symposium, San Diego(CA), 11-16 August, 2002.

[23] M. van Thiel and F.H. Ree. Phys. Rev. B, 48(6):3591, 1993.
[24] B. Leal Crouzet. PhD thesis, Université de Poitiers, 1998.

[25] M. van Thiel, F.H. Ree, and L.C. HaselMAN. report LLNL, UCRL-ID-120096, 1995.

[26] R. Meyer. Explosives. Verlag Chemie, Second Edition, 1981.

[27] D. PRICE. report Naval Surface Weapons Center, TR 82-298, 1982.

[28] E. N. Aleksandrov, V. A. Veretennikov, A. N. Dremin, and K.K. Shvedov. Combustion, Explosion, and Shock Waves, 3(4):471, 1967.

[29] J. Thouvenin. Détonique. CEA, 1997. 